\title{
Direct-to-consumer genome testing: opportunities for pharmacogenomics research?
}

This commentary examines the role that commercial providers of SNP-based genome-wide personalized risk profiles play in facilitating pharmacogenomics research. We first take a look at how personal genomics services, exemplified by the company 23 andMe, communicate information on drug response to customers. We then discuss the most important benefits and issues we see arising with the idea of 'crowdsourcing' pharmacogenomics research via commercial genome-scan providers. We conclude with a brief vision for the future.

\section{KEYWORDS: crowdsourcing ethics personal genomics pharmacogenomics testing}

\section{Pharmacogenomics \& direct-to-consumer genome testing: past \& present}

The field of direct-to-consumer genome testing is very young. Since autumn 2007, commercial companies have been offering individual genetic risk calculations over the internet. For fees starting at US $\$ 399$ these companies look at over 1 million SNP across the genome to assess their customers' individual genetic predispositions to various diseases, traits, carrier status and drug responses [1]. Customers go online to order a 'spit kit', fill it with saliva, send it back to the company and receive a password enabling them to access their personalized risk information online a few weeks later. The Reykjavík-based genome testing service deCODEme (Iceland) and 23andMe in Mountain View (CA, USA), were the first to offer these services direct-to-consumer on the internet. Navigenics (CA, USA) joined a few weeks later, and Pathway Genomics in San Diego (CA, USA) became the fourth personal genomics (PG) company to offer SNP-based genome-wide risk predictions to consumers online in summer 2009. With the exception of Navigenics, who restrict the scope of their tests to "important health conditions" and do not include testing for ancestry and drug response [101], all PG companies offer personalized risk calculations for a wide range of medical and 'recreational' phenotypes and traits (e.g., diabetes, alcohol flush syndrome and eye color), as well as results of SNP-based analysis of carrier status and drug response. Although drug response only accounts for a very small minority $(\leq 8)$ of the approximately 100 phenotypes tested by some PG companies, according to the co-founder of 23andMe, Anne Wojcicki, the possibility to test her child's genome before administering medication was one of the driving factors behind setting up her company [102]. Thus, pharmacogenomics testing is closely linked to the emergence of PG.

In this short commentary we will first take a look at how PG services, exemplified by 23 andMe, communicate information on genotype-determined drug-response phenotypes to customers. Subsequently, we will discuss the most important benefits and issues we see arising with the emergence of PG in this context. We will conclude with a vision for the future.

At the time of writing, one of the eight drug response-related characteristics that 23andMe tests for is abacavir hypersensitivity. The website offers an overview of the effect of the HIV drug abacavir in simple language, including links to further information, as well as to the scientific papers underpinning the risk calculations of the company [2-4]. Consumers also learn that " $8 \%$ of those treated with abacavir experience a hypersensitivity reaction characterized by fever, rash, respiratory problems, fatigue and nausea" [103]. By clicking on the "your genetic data' button, test-takers can learn whether or not they are likely to have a copy of the $H L A$ $B^{*} 5701$ variant and therefore whether they are at "greatly increased risk", or whether they have "no risk", for abacavir hypersensitivity. In
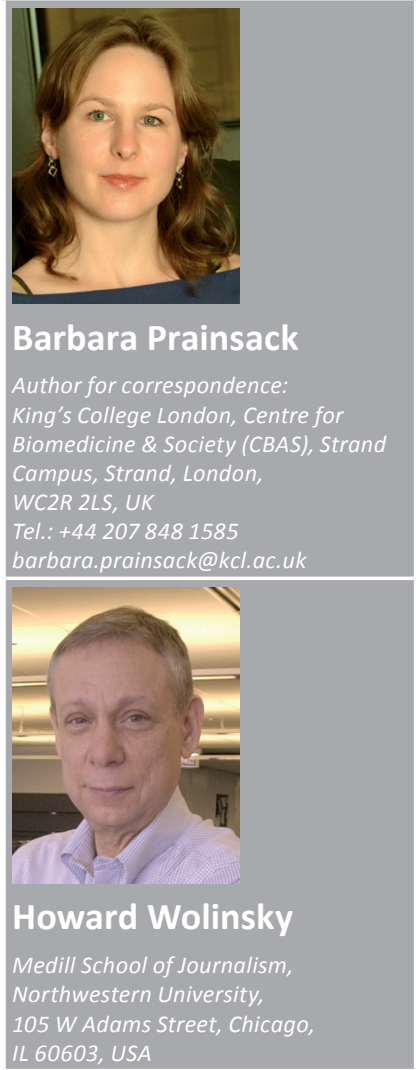
the same section customers are informed that the findings are only reliable for individuals of European ancestry, and that " $[\mathrm{t}]$ his SNP is a somewhat less reliable proxy for this marker in people from other ethnic groups" (this latter aspect reflects a key issue in current pharmacogenetics knowledge: unless more studies are carried out with participants with backgrounds other than European, the healthcare gap between Europeans and non-Europeans is likely to increase) [5]. Moreover, customers are also reminded that nongenetic factors can influence their sensitivity to abacavir.

Thus, educating customers regarding the importance of genetic variation for drug response is one role that the emerging PG industry can be seen to play. As commentators have pointed out, in order to help realize the potential of pharmacogenomics, education is crucial [6,7]. As if to illustrate this point, a 2008 survey of over 10,000 physicians carried out by the American Medical Association (IL, USA), in collaboration with the pharmacy benefits manager Medco Health Solutions Inc. (NJ, USA) found that while $98 \%$ of all respondents agreed a patient's genetic profile may influence drug therapy, only $10 \%$ felt that they were adequately informed regarding pharmacogenomic testing. Furthermore, only $26 \%$ had received some form of formalized pharmacogenomic training in medical school or during postgraduate training. Not surprisingly, those who said they were well informed were twice as likely to order pharmacogenomic tests as those who were not [104]. While no data on service uptake is available from other PG companies, 23 andMe recently announced to have " 30,000 active genomes" [105]. It can be expected that these consumers will function as multipliers to increase awareness of pharmacogenomics, a trend that could be facilitated further by initiatives of PG companies to 'educate' medical professionals [106,107] and the extensive media coverage that the emerging PG industry receives (FIGURE 1).

However, the PG industry does much more than providing information and education (one should not forget that those who have a stake in selling a product are usually not entirely unbiased in describing its value and importance) [1]. PG is also changing the way that research is carried out. Among PG companies, 23andMe pioneers this new approach: customers are encouraged not only to purchase a genome scan but to upload phenotypic information and lifestyle data also. For a few months in 2009, the company offered PG tests at a drastically reduced price (US\$99 instead of the full price of US\$399) to individuals who agreed to fill in a number of surveys and have their data passed on to academic and commercial disease research [108]. While this cheaper test did not include information on ancestry, carrier status or drug response, the media coverage surrounding it may have helped what arguably is an underlying idea of 23andMe's business model: to build a database comprising of genotypic, phenotypic and lifestyle information of a large number of individuals who are internet savvy and thus easily accessible for follow-up research; who have an apparent interest in health; and who are probably wealthy enough, on average, to pay for new diagnostic or therapeutic applications that this kind of research will generate. If this model works [109-111], then it is very likely to also be a feasible platform for certain areas of pharmacogenomics research. The reasons why PG companies have not made pharmacogenomics research a priority so far, despite the fact that it is exactly in this sector where their test results might prove most immediately actionable, are unclear [8]. Whether the approach to 'crowdsourcing' research will eventually prove successful, however, will depend on whether or not PG companies will manage to attract sufficiently large numbers of customers with the 'right' genotypes, and the willingness to make phenotypic and lifestyle information available. This, in turn, will hinge on how attractive an option participating in PG testing or research will be for consumers, both in terms of cost, and in terms of nonfinancial benefits. Representatives of the PG industry have already started criticizing publicly funded genomics research for not communicating research findings back to research participants $[112,113]$. It is likely that such discussions will affect what research participants expect as their return of investment from projects they volunteer for.

\section{Possible futures}

How feasible the idea to facilitate pharmacogenomics research via PG testing services is will ultimately also depend on the industry's assessment of what consumers want. Right from the start, PG companies have been very responsive to their customers desires and suggestions, as they were articulated on blogs or online publications. Also, 23andMe's decision in November 2009 to offer separate tests for ancestry and 
clinical reports

\begin{tabular}{|c|c|c|}
\hline Show results for Howard Wolinsky $:$ & & of elevated risks \\
\hline « Return to Overview | Disease Risks | Carrier Status | Traits | Drug Response & Recently Updated & \\
\hline Name & Status $\mathrm{V}$ & Last Updated \\
\hline Clopidogrel (Plavix@) Efficacy & Reduced & May 7, 2009 \\
\hline Abacavir Hypersensitivity new & Typical & Oct 8,2009 \\
\hline Fluorouracil Toxicity new & Typical & Oct 1,2009 \\
\hline Pseudocholinesterase Deficiency new & Typical & Nov 19, 2009 \\
\hline Warfarin (Coumadin@) Sensitivity & Typical & Mar 19, 2009 \\
\hline
\end{tabular}

The genotyping services of 23andMe are performed in LabCorp's CLIA-certified laboratory. The tests have not been cleared or approved by the FDA but have been analytically validated according to CLIA standards.

Figure 1. Personal genomics test result on genetic predisposition to drug response as provided by 23 andMe. (c) 23andMe, Inc. 2010. All rights reserved; distributed pursuant to a limited license from 23andMe.

disease-risk information, instead of marketing both in one package, is to be interpreted in this light. According to Stewart Ellis, General Manager of Ancestry Services at 23andMe, the company had recognized that there were two different markets, one for health and one for ancestry. Although Ellis finds it difficult to judge which of the two markets is larger, he deems customers interested in ancestry the far more vocal and active community (Stewart Ellis, interview with Howard Wolinsky, 3 December 2009). The company's decision to offer to people who are "not interested in the health stuff" [PG testtaker and Prainsack B, Pers. Comm.] an improved service at a price which is still competitive, will arguably allow the company to keep increasing their customer base and, thereby, the amount of data in their database potentially available for research. Ellis is quite explicit about the company's plans in the disease and pharmacogenomics research realm: 23 andMe expects to play a growing role in the future of facilitating "new discoveries regarding which drugs, what doses, both for prescription and over-the-counter medication" (Stewart Ellis, interview with Howard Wolinsky, 3 December 2009).

But what do consumers think? For example, one of us (Howard Wolinsky) was relieved to find that his genetic make-up was consistent with a medication he is taking (clopidogrel). Others might find information on their status as slow or rapid metabolizers, or their increased risk for drug toxicity, helpful in deciding (together with their treating physicians) which drugs to opt for. Based on conversations with test takers we assume that those interested in a PG test will not be deterred by legal restrictions: Testees told one of us (Howard Wolinsky) that the tough stance in New York, USA, has resulted in some testing fans, even for ancestry information, to take ferries over to or to drive to New Jersey or other states to use the US Postal Service or other delivery companies, this was done so that they are mailing their vials in from other states, which complies with the law. The results come in over the internet.

We expect that when SNP-based genome testing is succeeded by full sequences, the attractiveness to customers of both obtaining drug-relevant information from PG tests and the idea that PG services could become facilitators of pharmacogenomic research will increase. The more robust and comprehensive the genetic information on drug metabolism is, and the more easily this information is linked with other relevant information such as medical records and lifestyle information, 
the more clinically useful it will become $[9,10]$. For pharmacogenomics research specifically, the usefulness of the data will also depend on an increase of knowledge regarding the relationship between genotype-drug response and phenotype correlations. However, we predict that the availability of affordable full-genome sequences will take longer than commonly expected [11]. Moreover, we should not just blindly embrace the possibility of crowdsourcing pharmacogenomics research via commercial PG service providers, but also be aware of the fact that there is a trade-off between potential benefits for decision-making and privacy. Already at present, information of all customers of PG services may be passed on to law enforcement authorities, and in the case of some services, to other third parties who carry out research with the 'anonymized' data. It is not as much a particularity of genetic data as it is a particularity of the era of the internet that, once data is available online, it can never be removed entirely, and there is no absolute control over its proliferation. Control over one's health decision-making can come at the cost of control over one's data. How desirable a goal the privacy of genetic data is, and should be, however, is a different story [12].

\section{Acknowledgements}

The authors thank David Gurwitz for helpful comments on an earlier version of this manuscript.

\section{Financial \& competing interests disclosure}

The authors have no relevant affiliations or financial involvement with any organization or entity with a financial interest in or financial conflict with the subject matter or materials discussed in the manuscript. This includes employment, consultancies, honoraria, stock ownership or options, expert testimony, grants or patents received or pending, or royalties.

No writing assistance was utilized in the production of this manuscript.

\section{Bibliography}

Papers of special note have been highlighted as:

- of interest

" " of considerable interest

1 Prainsack B, Reardon J, Hindmarsh R, Gottweis H, Naue U, Lunshof JE: Misdirected Precaution. Nature 456, 34-35 (2008).

" Discusses regulatory challenges involved in direct-to-consumer genome testing and examines why instruments used to regulate 'traditional' clinical genetic testing do not fit this new field.

2 Mallal S, Phillips E, Carosi G et al:: $H L A-B^{*} 5701$ screening for hypersensitivity to abacavir. N. Engl. J. Med. 358(6), 568-579 (2008).

3 Colombo S, Rauch A, Rotger M et al.: The HCP5 single-nucleotide polymorphism: a simple screening tool for prediction of hypersensitivity reaction to abacavir. J. Infect. Dis. 198(6), 864-867 (2008).

4 Martin AM, Nolan D, Gaudieri S et al.: Predisposition to abacavir hypersensitivity conferred by $H L A-B^{*} 5701$ and a haplotypic Hsp70 Hom variant. Proc. Natl Acad. Sci. USA 101(12), 4180-4185 (2004).

5 Need AC, Goldstein DB: Next generation disparities in human genomics: concerns and remedies. Trends Genet. 25(11), 489-494 (2009).

" Critiques the under-representation of non-Caucasian groups in genetics and genomics research.
6 Frueh FW, Gurwitz D: From pharmacogenetics to personalized medicine: a vital need for educating health professionals and the community. Pharmacogenomics 5(5), 571-579 (2004).

7 Gurwitz D, Lunshof JE, Dedoussis G et al.: Pharmacogenomics education: International Society of Pharmacogenomics recommendations for medical, pharmaceutical, and health schools Deans of Education. Pharmacogenomics J. 5(4), 221-225 (2005).

8 Ng PC, Murray SS, Levy S, Venter JC: An agenda for personalized medicine. Nature 461, 724-726 (2009).

=- Discusses issues in communicating results of SNP-based genome-wide personalized risk profiles to consumers and recommends strategies for improving the utility of test results.

9 Steinbrook MD: Opportunities and Challenges for the NIH - an Interview with Francis Collins. N. Engl. J. Med. 361(14), 1321-1323 (2009).

10 Allison M: Can web 2.0 reboot clinical trials? Nat. Biotechnol. 27, 895-902 (2010).

11 Metzker ML: Sequencing technologies - the next generation. Nat. Rev. Genet. 11, 31-46 (2010).

- Offers a technical review of next-generation sequencing technologies.

12 Angrist M: Eyes wide open: the personal genome project, citizen science and veracity in informed consent. Per. Med. 6(6), 691-699 (2009).
-11. Discusses aspects of data privacy, confidentiality, and informed consent in connection with population genomics research.

\section{Websites}

101 Navigenics

www.navigenics.com/visitor/what_we_offer (Accessed 15 November 2009).

102 Shiels M: Valley Girls: Linda Avey and Anne Wojcicki (20 November 2009)

http://news.bbc.co.uk/2/hi/ technology/7738768.stm (Accessed 22 December 2009)

10323 andMe website on drug abacavir hypersensitivity www.23andme.com/you/journal/ abacavir/overview (Accessed 15 November 2009)

104 Stanek EJ, Sanders CL, Teagarden JR et al.: National Pharmacogenomics Physician Survey: who are the physicians adopting pharmacogenomics and how does knowledge impact adoption? Abstracts and summaries (2009)

www.medcoresearch.com/community/ pharmacogenomics/physicansurvey (Accessed 15 November 2009)

105 TEDMED: 23andMe has 30,000 'active' genomes, launching 'Relative Finder' soon (28 October 2009) http://mobihealthnews.com/5131/ 23andme-has-30000-active-genomeslaunching-relative-finder-soon (Accessed 15 November 2009) 
106 Henderson M: Genetic testing company 23andme may offer GPs a chance to try service (15 September 2009)

www.timesonline.co.uk/tol/news/science/ genetics/article6834143.ece

(Accessed 15 November 2009)

107 Navigenics press release (21 October 2009) www.navigenics.com/visitor/about_us/press/ releases/bidmc_102109

(Accessed 15 November 2009).

108 Kincaid J: 23andMe Launches $\$ 99$ Kit To Spur Its 'Research Revolution' (8 July 2009). Tech Crunch website www.techcrunch.com/2009/07/08/ 23andme-launches-99-kit-to-spur-itsresearch-revolution (Accessed 15 November 2009)

109 MacArthur D: 23andMe launches new effort to recruit patients for disease gene studies. Genetic Future blog (7 July 2009) http://scienceblogs.com/

geneticfuture/2009/07/23andme_launches_ new_effort_to.php

(Accessed 15 November 2009)

110 MacArthur D: 23andMe presents novel genetic associations at American Society of Human Genetics meeting. Genetic Future blog (25 October 2009)

http://scienceblogs.com/

geneticfuture/2009/10/23andme_presents_ novel_genetic.php

(Accessed 15 November 2009).

111 Vorhaus D: Genomic Research Continues to Go DTC (12 November 2009) Genomics Law Report www.genomicslawreport.com/index. php/2009/11/12/genomic-research-continuesto-go-dtc

(Accessed 15 November 2009)
112 Vorhaus D: Is there an obligation to return genetic data to research participants? Kaiser Responds to 23andMe's TEDMED criticism. (3 November 2009) Genomics Law Report www.genomicslawreport.com/index. php/2009/11/03/kaiser-23andme-tedmed (Accessed 15 November 2009)

113 McCarty CA: To share or not to share: that is the question. (Commentary in the Genomics Law Report's series 'What ELSI is New'?) (2009)

www.genomicslawreport.com/index. php/2009/10/15/to-share-or-not-to-sharethat-is-the-question

(Accessed 15 November 2009) 